\title{
What We Know About Diet, Genes, and Dyslipidemia: Is There Potential for Translation?
}

\author{
Toni I. Pollin • Michael Quartuccio
}

Published online: 15 October 2013

(C) Springer Science+Business Media New York 2013

\begin{abstract}
Cardiovascular disease, particularly coronary artery disease (CAD), is the leading cause of death in the United States. Dyslipidemia, including elevated low density lipoprotein cholesterol (LDL-C) and triglyceride (TG) levels and low high-density lipoprotein cholesterol (HDL-C), is a wellestablished risk factor for CAD and is influenced by both genetic and lifestyle factors, including the diet and dietary fat in particular. Major strides in elucidating the genetic basis for dyslipidemia have been made in recent years, and the quest to clarify how genetic differences influence lipid response to dietary intervention continues. Some monogenic conditions such as famililal hypercholesterolemia and sitosterolemia already have customized dietary recommendations. Some promising associations have emerged for more polygenic dyslipidemia; but further studies are needed in large dietary intervention studies capturing increasing amounts of explainable genetic variation before recommendations can be made for clinical translation.
\end{abstract}

Keywords Genetics · Nutrigenomics · Gene-diet interactions · Cholesterol · Dietary fat · Triglycerides · Monounsaturated fat · Polyunsaturated fat · Dietary intervention studies

T. I. Pollin · M. Quartuccio

Department of Medicine, University of Maryland School of

Medicine, Baltimore, MD, USA

T. I. Pollin

Program in Personalized and Genomic Medicine, Department of Epidemiology \& Public Health, University of Maryland School of Medicine, Baltimore, MD, USA

T. I. Pollin $(\bowtie)$

University of Maryland School of Medicine, 660 West Redwood

Street, Room 445C, Baltimore, MD 21201, USA

e-mail: tpollin@medicine.umaryland.edu

\section{Introduction}

Cardiovascular disease (CVD), in particular coronary artery disease (CAD), is the leading cause of death in the United States [1]. Dyslipidemia, comprising elevated serum lowdensity lipoprotein cholesterol (LDL-C) and/or triglyceride (TG) levels and/or decreased high-density lipoprotein cholesterol (HDL-C) levels, is an established risk factor for CAD [2]. More recently, it has been possible to measure more specific traits underlying circulating cholesterol and TG levels such as lipoprotein particle size and number, but using such traits to predict cardiovascular risk is not currently standard of care [3••]. Dyslipidemia has long been known to have a strong genetic basis [4], and many of the specific variants have been identified, including at least 95 specific genetic variants that modify lipid levels in the general population [5••] along with numerous monogenic/high penetrance dyslipidemia syndromes [6]. Lipid levels are also modifiable through changes in diet (e.g., reduction in dietary fat and calories), physical activity and weight $[7,8]$. Since both genes and lifestyle factors including diet influence circulating lipid levels, it stands to reason that dietary changes might affect individuals differently depending upon their genotype and that dietary recommendations tailored to genotype might produce more efficacious results. Some rare forms of monogenic dyslipidemia are associated with specific dietary prescriptions. These can be such as a diet low in saturated fat and cholesterol in individuals with familial hypercholesterolemia due to a highly penetrant $L D L R$ (LDL receptor) or $A P O B$ mutation [9॰] or avoidance of margarines containing stanols in individuals with homozygous sitosterolemia [10]. This is a rare condition involving hyper-absorption of plant sterols in which cholesterol is often elevated. The latter case is particularly illustrative of the potential importance of genotype for dietary considerations because stanol-containing margarines have otherwise been recommended for managing hypercholesterolemia [10]. 
However, there are currently no consensus guidelines on the use of information about more common genetic variations to guide dietary recommendations, though studies of gene $\mathrm{x}$ diet interactions have been conducted for several decades (systematically reviewed by Masson et al. (2003) [11]). In this review, we discuss studies conducted during the past year, some of which have attempted to address limitations in sample size and study design of previous studies and have begun to look more closely at specific types of dietary fat.

\section{Dietary Fat as Modifier of the Effect of Genetic Polymorphisms on Lipid Levels}

Most studies evaluating gene $\mathrm{x}$ diet interactions in the context of lipids focus on the ability of alterations in consumption of total fat, saturated fat and, in some cases, monounsaturated fatty acids (MUFA) and polyunsaturated fatty acids (PUFA) to modulate the relationship between specific candidate genes and lipid levels. The following are summaries categorized by the particular lipid phenotype in which an interaction was studied.

\section{Triglycerides (TG)}

$A P O E$ encodes apolipoprotein E (APOE), a polymorphic apolipoprotein involved in regulation of lipoprotein metabolism through modulation of the affinity of lipoprotein particles for the low density lipoprotein (LDL) receptor. The three major isoforms of $A P O E$ (APOE2, APOE3 and APOE4) are defined by two single nucleotide coding polymorphisms, rs7412 (Arg158Cys) and rs429358 (Cys130Arg). The APOE4 allele, in addition to its association with increased risk for Alzheimer disease [12], is also associated with increased plasma LDL-cholesterol and triglycerides and risk for coronary heart disease [13]. Numerous studies examining the interaction of $A P O E$ genotype with dietary fat and cholesterol have been published since 1983 [14] (systematically reviewed in 2003 [11] and reviewed in 2007 [15]) showing evidence that APOE4 carriers have a greater sensitivity to cholesterolraising effects of dietary fat and cholesterol in some but not all studies $[11,15]$. A couple of previous studies provided evidence that fish oil, often used as a strategy for lowering TGs in those with diets high in saturated fats, may have greater consequent LDL-C-raising effects in APOE4 carriers [16, 17]. Carvalho-Wells et al. (2012) [18•] used a prospective genotype-based recruitment design to look specifically at the interactive effects of fish oil, or docosahexaenoic acid (DHA) and saturated fatty acids (SFA) [18•] on lipid levels. They recruited 90 individuals in which an equal number of individuals with the APOE4/APOE3 genotype (APOE4 carriers, normally comprising $20-25 \%$ of Caucasian individuals) for a three-diet crossover study. There was no gene $\mathrm{x}$ diet interaction on the primary endpoint of LDL-C levels, thus contradicting these authors' previous evidence of increased LDL-C raising with DHA supplementation in APOE4 carriers [16, 17], a discrepancy that may be due to differences in DHA dose, lipid profile and habitual fat intake [18•]. Only TGs showed significant evidence for gene $\mathrm{x}$ diet interaction $(p=$ 0.03). APOE4 carriers had higher TGs than APOE3 homozygotes on both a low fat ( $28 \%$ fat, $8 \%$ saturated fat) and high fat diet (38\% fat, $18 \%$ saturated fat) but similar TG levels when the high fat diet was supplemented with 3.45 grams/day DHA fish oil. These findings suggest that fish oil may be particularly efficacious at lowering TG in APOE4 carriers, or in ameliorating the APOE4-related TG elevation. The authors also suggest that some of the discrepancy among studies regarding APOE $\mathrm{x}$ dietary fat consumption may relate to whether or not APOE4 homozygotes (comprising only 1$2 \%$ of the general population) are included (which they were not in this study) as they may be particularly sensitive to dietary fat $[18 \bullet]$.

Another recent gene $\mathrm{x}$ diet study of APOE variation published this year utilized a cross-sectional study design with dietary recall in 996 Lithuanian individuals and found no significant interaction of APOE2 and APOE4 alleles with a food frequency questionnaire-based on fat intake on TG [19].

PER2 encodes Period 2, a transcription factor involved in circadian regulation, and was selected for study because of previous work demonstrating coordination with factors involved in lipid metabolism [20] and association of PER2 and other clock gene variation/expression with metabolic syndrome features in both mice, including with response to dietary fat modulation [21], and humans [22, 23]. While dietary intake was not altered or recorded, circulating polyunsaturated fatty acids (PUFA), monounsaturated fatty acids (MUFA) and saturated fatty acids (SFA), were each measured and calculated as a percent of total lipids in 381 European individuals with metabolic syndrome in the LIPGENE study. The rs $2304672 \mathrm{G}$ allele was associated with increased TGrich lipoprotein TG (TRL-TG) $(p=0.009)$ and apoC-III $(p=$ 0.033 ). When participants were stratified by median circulating \%SFA, the increased TRL-TG and apoC-III became more significant in the high \%SFA $(\geq 30.9 \mathrm{mmol} / \mathrm{L})$ group $(p<$ 0.001 and $p=0.009$, respectively), and significantly higher levels of TG $(p=0.001)$, apoB-48 $(p=0.028)$, and apoC-II $(p=0.001)$ were also seen in $\mathrm{G}$ carriers in the high \%SFA group. These associations were not seen in the low \%SFA group, and interactions were significant for most with $p$ values ranging from 0.046 to $<0.001$. While this was not a dietary study, further studies to assess whether this polymorphism alters the relationship between saturated fat intake and TG homeostasis would be of interest.

PPARG encodes peroxisome proliferator-activated receptor gamma, a transcription factor regulating expression of 
genes involved in lipid metabolism, inflammation and adipose cell differentiation. The common PPARG polymorphism ProAla12 was studied in the Reading Imperial Survey Cambridge King's (RISCK) study, in which 549 subjects completed a study in which they were randomized to diets high in saturated fat (HS), high in MUFA (HM) or low in fat and high in carbohydrates (LF) [24]. At recruitment Ala12 carriers showed evidence for association lower TG with higher PUFA:SFA (P:S) ratio at ratios above $0.34(p=0.002)$, whereas TG did not vary across P:S ratio quartiles in Pro12 homozygotes (interaction $p=0.02$ ). The HM and LF diets did not reduce TGs and no interaction of Pro12Ala or the PPARA Val162Leu polymorphism with diet on TG was seen [24].

$A B C G 8$, discussed more extensively below in the context of LDL-C and total cholesterol, encodes a half ATP transporter, which along with the $A B C G 5$ product, limits the absorption of plant sterols (PS) from the intestine and facilitates the excretion of PS through the bile. In the study reported below by Myrie et al. (2012) [25•], a significant genotype group $x$ plant sterol treatment effect was seen when comparing individuals heterozygous for a loss of function mutation in $A B C G 8$ with their non-carrier relatives with respect to $\mathrm{TG}$ levels. However, the treatment did not alter TG levels in either group and the interaction term was found to result from higher TG levels in the control subjects (possibly by chance) and not to provide information useful for using genotype to customize dietary intervention [25•].

The FADS gene cluster (FADS1, FADS2, and FADS3) as discussed below with respect to LDL-C and TC, showed evidence for association with TG levels in 2006 German children, but analyses of gene $\mathrm{x} \omega-3$ PUFA dietary content on TG levels were negative [26]. In the Malmö Diet and Cancer cohort, also discussed below, the variant tested was neither associated with TG nor modified by PUFA intake on TG [27].

\section{LDL-Cholesterol and/or Total Cholesterol (TC)}

$A P O A 5$ encodes apolipoprotein $\mathrm{A} 5$, a component of the APOA1-APOC3-APOA4-APOA5 cluster on chromosome $11 \mathrm{q} 23$. The polymorphism rs 964184 , located $11 \mathrm{~kb}$ upstream of the $A P O A 5$ gene and in the 5' untranslated region of a zinc finger protein, was selected for study because of its robust association with multiple lipid traits in several large genomewide association and meta-analysis studies of lipid traits [5••]. Seven hundred thirty-four participants in the Pounds Lost Trial, a two year randomized trial of diets with four different fat/protein/carbohydrate compositions, were genotyped for rs964184 [28••]. The G risk allele, which was not associated with LDL-C or total cholesterol levels at baseline, showed enhanced TC $(\beta=-9.3 \pm 4.4 \mathrm{mg} / \mathrm{dl}$ greater reduction, $p=0.036)$ and LDL $(\beta=-7.5 \pm 3.6 \mathrm{mg} / \mathrm{dl}$ greater reduction, $p=0.039)$ lowering in response to a low fat diet (20\% fat) but no association with changes in these traits in the high fat diet
( $40 \%$ fat) group. Interactions were significant at $p=0.007$ and 0.017 for TC and LDL-C, respectively, $p=0.0005$ and $p=$ 0.002 for linear mixed models incorporating six month values in addition to baseline and two years [28••]. With replication of these robust findings, this or other variants in or near $A P O A 5$ and the cluster may prove to be useful targets for intensifying lowering of dietary fat.

$A P O E$, as discussed above, showed evidence of interaction with DHA supplementation to a high fat diet on TG levels in a prospective study [18•], but in neither the prospective study nor the cross-section study of Lithuanians [19] was evidence of gene $\mathrm{x}$ fat intake effect seen on LDL-C or TC.

The FADS gene cluster (FADS1, FADS2, and FADS3) encodes fatty acid saturases and variation within it was previously associated with lipid levels [5••]. The gene products are known to be key enzymes in the conversion of $\alpha$-linoleic acid (ALA) and linoleic acid (LA) into long chain PUFAs. Therefore, Hellstrand et al. [27] studied whether long chain $\omega-3$ PUFA intake (based on dietary questionnaires, diaries and interviews) would modulate the association of the FADS1 rs 174547 polymorphism with lipid levels in 4,635 healthy participants in the Malmö Diet and Cancer (MDC) cohort. They found that the rs $174547 \mathrm{C}$ allele was associated with lower LDL-C levels overall $(p=0.03)$. When participants were stratified into tertiles of long chain $\omega$-3 PUFA intake, the variant was associated with LDL-C more significantly $\left(p=2 \times 10^{-4}\right)$ in the low and not significantly associated in the medium and high intake tertiles, suggesting that FADS gene associations with lipid levels are modifiable by alterations in PUFA intake [27], but as noted by the authors, requiring replication in well-powered studies with high quality dietary data in order to inform future intervention strategies.

Several of the SNPs in the FADS cluster, including rs174547 and five additional SNPs highly correlated with one another, were also studied for interactions with $\omega$-3 PUFA intake as assessed by food frequency questionnaire on lipids in 2006 German children participating in two cohort studies [26]. Both PUFA intake and FADS variants were associated with LDL-C and TC levels, but significant interactions were not observed. The data was not shown for the interactions, so it could not be surmised whether there was any non-significant suggestion of modification of the genetic association by PUFA intake as in the MDC cohort [26].

PPARG Pro12Ala showed evidence in the RISCK Study [24] of interaction with a P:S ratio on LDL-C $(p=0.002)$. When combined with the PPARA Leu162Val genotype, an interaction was seen whereby individuals carrying both the Ala12 and Val162 alleles had differential LDL-C responses to the HM and LF diets, with higher LDL-C and small dense LDL proportion in that genotype group. The other genotype groups did not show differences in LDL-C and sdLDL between the $\mathrm{HM}$ and LF diets. Interactions were significant at $p=$ 0.003 and $p=0.012$ for LDL-C and sdLDL, respectively [24]. 
$K D R$ encodes the kinase insert domain receptor also known as the vascular endothelial growth factor receptor-2 (VEGFR2). Yap et al. [29] performed factor analysis on the dietary patterns of 136 Japanese individuals and separately in 179 Chinese Malaysian individuals. They then examined tertiles of factor scores for Western vs. Japanese diet in the Japanese sample and Balanced Diet vs. Meat, Rice and Noodles Diet in the Chinese Malaysian sample, and they genotyped one common known functional coding variant (rs1870377, Gln472His) and one common known functional regulatory variant (rs2071559) in the two samples. In the Japanese sample, a significant interaction was observed between Western diet tertile (T2+T3 vs. T1) and rs2071559 genotype (TT vs. CC+CT) such that in T1, the TT genotype was associated with lower LDL-C, while in T2+ T3, the TT genotype was associated with higher LDL-C ( $p=$ 0.016 for interaction). In the Chinese Malaysian sample, the rs1870377 TT vs. AA+AT genotype was associated with higher LDL-C in the highest Meat/rice/noodles diet tertile, but not in the other tertiles ( $p<0.001$ for interaction) [29].

$A B C G 8$ encodes a half ATP transporter, which along with ABCG5, limits the absorption of plant sterols (PS) from the intestine and facilitates the excretion of PS through the bile. There is evidence that in normal individuals, ingestion of PS reduces CVD risk by lowering lipid levels. Individuals homozygous for loss of function mutations in the $A B C G 5$ or $A B C G 8$ gene have a condition known as sitosterolemia or phytosterolemia, which causes elevated levels of plant sterols and cholesterol and premature coronary artery disease. PS supplementation is contraindicated in sitosterolemia homozygotes because of the nature of the defect, but it was not known if this was the case for heterozygous carriers. Myrie et al. (2012) [25•] sought to determine whether heterozygosity for a loss of function mutation in $A B C G 8$ (S107X) would have a favorable or adverse response to PS supplementation and compared ten mutation carriers to 15 control subjects in a double blind crossover study of 1.6 grams of PS per day vs. a placebo. In both genotype groups, TC and LDL-C concentrations decreased in response to PS treatment. The decreases were similar in magnitude in both groups, with TC decreasing by $0.46 \pm 0.13(\mathrm{SEM}) \mathrm{mmol} / \mathrm{L}$ in the heterozygotes and $0.22 \pm$ $0.10 \mathrm{mmol} / \mathrm{L}$ in the non-carriers $(p=0.003$ for treatment effect, $p=0.75$ for group $\mathrm{x}$ treatment interaction) and LDL-C decreasing by $0.36 \pm 0.18 \mathrm{mmol} / \mathrm{L}$ in the heterozygotes and $0.32 \pm$ $0.10 \mathrm{mmol} / \mathrm{L}$ in non-carriers $(p=0.006$ for treatment effect, $p=0.40$ for group $\mathrm{x}$ treatment interaction) when subjects were treated with PS. Tracer assays were also used to show there were no differences in cholesterol absorption or efficiency. Since even $50 \%$ ABCG8 activity appears sufficient for individuals to reap the benefits of PS supplementation, these results suggest that PS supplementation is only contraindicated in cases of absolute transporter deficiency [25•].

A genetic risk score (GRS) consisting of 32 polymorphisms previously associated with lipid levels was studied by one of the authors of this review (TIP) and colleagues for interaction with dietary and metformin interventions in 2,993 individuals in the Diabetes Prevention Program (DPP) [30]. The lifestyle intervention consisted of $150 \mathrm{~min} / \mathrm{wk}$ of physical activity and a goal of loss of $7 \%$ of baseline weigh through a focus on daily fat reduction. This study evaluated gene $\mathrm{x}$ treatment interactions not only on standard lipid levels but also on several nuclear magnetic resonance derived lipoprotein sub-fraction traits. Significant interactions of the lifestyle intervention with GRS were seen on LDL-C levels as well as small LDL particles, whereby a GRS was associated with higher baseline-adjusted one year LDL-C $\left(p=8 \times 10^{-5}\right)$ and higher density of small LDL particles $(p=0.01)$ in the lifestyle group but not in the placebo group ( $p=0.35$ and 0.74 for LDL$\mathrm{C}$ and small LDL particle density respectively; interaction $p=$ 0.02 and 0.01 respectively). Because the DPP intervention incorporated increased physical activity as well as total caloric and fat reduction, the effect modification could not be traced specifically to dietary fat reduction, nor could the interaction be traced to a specific genetic marker. Nonetheless, these findings suggested that those with higher genetic loading for dyslipidemia are less responsive to a dietary/activity intervention for lowering LDL-C and small LDL particle levels and may need to be targeted with a more aggressive lifestyle and/ or pharmaceutical intervention [30].

\section{HDL-Cholesterol}

$A P O A 5$, discussed in the LDL-C section as a modulator of low fat diet response in the Pounds Lost Trial, also showed evidence of interaction with HDL-C response to a high (40\%) fat diet. The rs964184-G allele was associated with a greater increases in HDL-C in the high fat diet group ( $p=0.038$ ), with evidence for gene $\mathrm{x}$ diet interaction at $p=0.006$ [28••].

FADS1 SNP rs174547 as studied in 4,635 MDC cohort participants [27] as discussed above with respect to TG, was not associated with HDL-C levels at baseline. However, when stratified by $\alpha$-linoleic acid/linoleic acid (ALA/LA) intake, an interaction emerged in which the previously described HDL-C lowering $\mathrm{C}$ allele was borderline $(p=0.07)$ associated with lower HDL-C levels in the low ALA/LA tertile but not the medium and high tertiles [27].

$C N R 1$ encodes the $\mathrm{G}$ protein-coupled receptor $\mathrm{CB}_{1}$, a component of the endocannabinoid system, which has previously shown effects on adiposity and lipoprotein metabolism. CNR1 haplotypes were studied in three dietary intervention cohorts for evidence of interaction with high fat (HF) vs. low fat (LF) diets comprising a total of 590 individuals [31 •]. A study of 411 normal weight males on eucaloric diets with $35 \%$ (HF) vs. $20 \%$ (LF) fat yielded modest evidence of interaction $(p=0.03)$ that was not considered significant due to multiple comparisons. In a similar study of 71 women, there was a greater HF/ LF HDL-C difference in those carrying a particular haplotype 


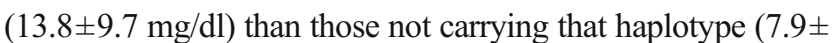
$6.2 \mathrm{mg} / \mathrm{dl})$, with significant evidence of interaction $(p=0.007)$. A third cohort of 108 obese women showed that carriers had higher HDL-C levels than non-carriers during the HF diet (52.3 vs. $45.5 \mathrm{mg} / \mathrm{dl} p=0.009)$ but not on the low fat diet (50.1 vs. $48.0 \mathrm{mg} / \mathrm{dl}, p=0.41$ ); a $p$-value for the difference as in the first and second cohorts is not given [31•].

\section{Additional Studies}

Skeletal muscle expression of several lipid metabolism genes, including but not limited to the peroxisome proliferator activated receptors PPAR $\alpha, \operatorname{PPAR} \delta$ and genes under their regulation was evaluated in 84 subjects with metabolic syndrome who were randomly assigned to one of four different diets: high-SFA (HSFA), high-MUFA (HMUFA), low-fat high complex carbohydrate (LFHCC) diet, or LHFCC supplemented with $\omega$-3 PUFA (LFHCCn-3) [32••]. Expression of SREBF1 (sterol regulatory element binding transcription factor 1, identified in the report by its alternative symbol SREBPIc, or sterol-regulatory element binding protein) showed HMUFAspecific increases in expression in men $(p=0.02)$, and expression of MLXIPL (MLX interacting protein-like, identified in the report by alternative alias $C h R E B P$, or carobohydrateresponsive binding element protein) showed a trend ( $p=$ $0.14)$ toward HSFA-specific increased expression in both men and women. Differential diet-specific gene expression responses of insulin-sensitive vs. insulin resistant subjects were shown for seven of 21 genes tested [32••]. While this study did not specifically look at dietary effects on particular genotypes, these findings may help to inform future attempts to assess genotype specific responses to dietary fat alteration.

A randomized controlled trial of the effect of polyphenolrich cloudy apple juice on obesity-related traits including lipids was conducted in 68 overweight to obese men and yielded decreases in body fat percent but no effect on lipids [33]. Gene $\mathrm{x}$ intervention analyses were conducted for variants in several candidate genes (FABP2, IL6, INSIG2, PPARGC1A [identified by alternative alias $P G C 1$ ], PPARG and $U C P 3$ ) with all traits and yielded evidence for interaction of the IL6 rs 1800795 polymorphism with body fat reduction but no gene $\mathrm{x}$ intervention effects on lipids [33]. Thus, this study was negative for either general or genotype-specific effects of polyphenol supplementation on lipid levels.

\section{Conclusions: Summary and Potential for Translation}

Given the challenges inherent in either controlling or accurately recording dietary intake in humans, combined with power issues involved in detecting a statistical interaction, finding robust interactions that point clearly to clinical translation has so far been largely elusive. Comprehensive characterization of the genomic architecture of circulating lipid levels [5••] is still in its infancy, with most causal variants not yet elucidated. Moreover, multiple comparisons issues introduced by evaluating large numbers of loci add additional power issues. Consequently, to date in most studies, dietary parameters as modifiers of the relationships between genetic variation and circulating lipid levels have been hypothesis-driven studies focused on particular candidate genes. Replication of some of the more robust findings, such as the modification of the dietary fat/ serum cholesterol by the APOA5 variant [28••] in wellcontrolled, adequately powered dietary intervention cohorts will be needed in order for translation to become a possibility. However, even in such situations, incorporating one individual common variant, even a known functional one, will leave a large amount of variance unexplained and lead to variation in outcomes and the often mixed results that have been observed in the past. This issue was addressed through the use of a multiSNP genomic risk score DPP [30], but the translatability of the DPP findings was limited by the known genetic architecture at the time of the analysis and the inability to separate effects of weight loss, overall caloric reduction and increases in physical activity from dietary fat reduction. As the genomic architecture of lipid variation continues to be elucidated beyond genomewide association studies toward whole genome and exome sequencing and functional validation, it will be important to study the ability of multiple functional genetic variants to simultaneously alter the relationship between intake of fat and other nutrients with lipid levels in well controlled adequately powered dietary intervention and cardiovascular outcome studies. This would enable the expansion of the pool of people who can benefit from personalized dietary intervention beyond those with highly penetrant forms of dyslipidemia.

\section{Compliance with Ethics Guidelines}

Conflict of Interest Toni I. Pollin and Michael Quartuccio declare that they have no conflict of interest.

Human and Animal Rights and Informed Consent This article does not contain any studies with human or animal subjects performed by any of the authors.

\section{References}

Papers of particular interest, published recently, have been highlighted as:

- Of importance

-. Of major importance

1. National Heart, Lung, and Blood Institute (NHLBI). Morbidity and Mortality: 2012 Chartbook on Cardiovascular, Lung, and Blood Diseases. Bethesda, MD : U.S. Department of Health and Human 
Services, Public Health Service (PHS), National Institutes of Health (NIH) ; 2012.

2. Third Report of the National Cholesterol Education Program (NCEP) Expert Panel on Detection, Evaluation, and Treatment of High Blood Cholesterol in Adults (Adult Treatment Panel III) final report. Circulation. 2002;106(25):3143-421.

3. •- Davidson MH, Ballantyne CM, Jacobson TA, et al. Clinical utility of inflammatory markers and advanced lipoprotein testing: advice from an expert panel of lipid specialists. J Clin Lipidol. 2011;5(5): 338-67. doi:10.1016/j.jacl.2011.07.005. This article helps to clarify the differences in information and utility between existing lipid screening tests and emerging markers.

4. Namboodiri KK, Kaplan EB, Heuch I, et al. The Collaborative Lipid Research Clinics Family Study: biological and cultural determinants of familial resemblance for plasma lipids and lipoproteins. Genet Epidemiol. 1985;2(3):227-54. doi:10.1002/gepi.1370020302.

5. •• Teslovich TM, Musunuru K, Smith AV, et al. Biological, clinical and population relevance of 95 loci for blood lipids. Nature. 2010;466(7307):707-13. doi:10.1038/nature09270. This article describes the results population in the largest genome-wide association meta-analysis study of genetic loci affecting lipid levels to date.

6. Hegele R. Monogenic dyslipidemias: window on determinants of plasma lipoprotein metabolism. Am J Hum Genet. 2001;69(6): 1161-77. doi: $10.1086 / 324647$.

7. Yu-Poth S, Zhao G, Etherton T, Naglak M, Jonnalagadda S, KrisEtherton PM. Effects of the National Cholesterol Education Program's Step I and Step II dietary intervention programs on cardiovascular disease risk factors: a meta-analysis. Am J Clin Nutr. 1999;69(4):632-46.

8. Kraus WE, Houmard JA, Duscha BD, et al. Effects of the amount and intensity of exercise on plasma lipoproteins. N Engl J Med. 2002;347(19):1483-92. doi:10.1056/NEJMoa020194.

9. - Descamps OS, Tenoutasse S, Stephenne X, et al. Management of familial hypercholesterolemia in children and young adults: consensus paper developed by a panel of lipidologists, cardiologists, paediatricians, nutritionists, gastroenterologists, general practitioners and a patient organization. Atherosclerosis. 2011;218(2):272-80. doi:10. 1016/j.atherosclerosis.2011.06.016. This article describes treatment and management recommendations, including dietary, for a classic monogenic lipid disorder.

10. Merkens LS, Myrie SB, Steiner RD, Mymin D. Sitosterolemia. In: Pagon RA, Adam MP, Bird TD, Dolan CR, Fong CT, Stephens K, editors. GeneReviews. Seattle: University of Washington; 1993.

11. Masson LF, McNeill G, Avenell A. Genetic variation and the lipid response to dietary intervention: a systematic review. Am J Clin Nutr. 2003;77(5):1098-111.

12. Statement on use of apolipoprotein $E$ testing for Alzheimer disease. American College of Medical Genetics/American Society of Human Genetics Working Group on ApoE and Alzheimer disease. JAMA. 1995;274(20):1627-29.

13. Song Y, Stampfer MJ, Liu S. Meta-analysis: apolipoprotein E genotypes and risk for coronary heart disease. Ann Intern Med. 2004;141(2):137-47.

14. Fisher EA, Blum CB, Zannis VI, Breslow JL. Independent effects of dietary saturated fat and cholesterol on plasma lipids, lipoproteins, and apolipoprotein E. J Lipid Res. 1983;24(8):1039-48.

15. Minihane AM, Jofre-Monseny L, Olano-Martin E, Rimbach G. ApoE genotype, cardiovascular risk and responsiveness to dietary fat manipulation. Proc Nutr Soc. 2007;66(2):183-97. doi:10.1017/ S0029665107005435.

16. Olano-Martin E, Anil E, Caslake MJ, et al. Contribution of apolipoprotein E genotype and docosahexaenoic acid to the LDL-cholesterol response to fish oil. Atherosclerosis. 2010;209(1):104-10. doi:10. 1016/j. atherosclerosis. 2009.08.024.

17. Minihane AM, Khan S, Leigh-Firbank EC, et al. ApoE polymorphism and fish oil supplementation in subjects with an atherogenic lipoprotein phenotype. Arterioscler Thromb Vasc Biol. 2000;20(8): 1990-7.

18. - Carvalho-Wells AL, Jackson KG, Lockyer S, Lovegrove JA, Minihane AM. APOE genotype influences triglyceride and Creactive protein responses to altered dietary fat intake in UK adults. Am J Clin Nutr. 2012;96(6):1447-53. doi:10.3945/ajen.112.043240. This article describes the most recent prospective study of many studies of APOE genotype and dietary fat response.

19. Petkeviciene J, Smalinskiene A, Luksiene DI, et al. Associations between apolipoprotein $\mathrm{E}$ genotype, diet, body mass index, and serum lipids in Lithuanian adult population. PLoS One. 2012;7(7): e41525. doi:10.1371/journal.pone.0041525.

20. Schmutz I, Ripperger JA, Baeriswyl-Aebischer S, Albrecht U. The mammalian clock component PERIOD2 coordinates circadian output by interaction with nuclear receptors. Genes Dev. 2010;24(4): 345-57. doi:10.1101/gad.564110.

21. Dallmann R, Weaver DR. Altered body mass regulation in male mPeriod mutant mice on high-fat diet. Chronobiol Int. 2010;27(6): 1317-28. doi:10.3109/07420528.2010.489166.

22. Hernandez-Morante JJ, Gomez-Santos C, Margareto J, et al. Influence of menopause on adipose tissue clock gene genotype and its relationship with metabolic syndrome in morbidly obese women. Age (Dordr). 2012;34(6):1369-80. doi:10.1007/s11357-011-9309-2.

23. Gomez-Abellan P, Hernandez-Morante JJ, Lujan JA, Madrid JA, Garaulet M. Clock genes are implicated in the human metabolic syndrome. Int J Obes (Lond). 2008;32(1):121-8. doi:10.1038/sj.ijo. 0803689.

24. AlSaleh A, Sanders TA, O'Dell SD. Effect of interaction between PPARG, PPARA and ADIPOQ gene variants and dietary fatty acids on plasma lipid profile and adiponectin concentration in a large intervention study. Proc Nutr Soc. 2012;71(1):141-53. doi:10.1017/ S0029665111003181.

25. • Myrie SB, Mymin D, Triggs-Raine B, Jones PJ. Serum lipids, plant sterols, and cholesterol kinetic responses to plant sterol supplementation in phytosterolemia heterozygotes and control individuals. Am J Clin Nutr. 2012;95(4):837-44. doi:10.3945/ajen.111.028985. This article describes a study seeking to determine whether the personalized dietary recommendations for those homozygous for mutations causing sitosterolemia might more broadly apply to heterozygotes.

26. Standl M, Lattka E, Stach B, et al. FADS1 FADS2 gene cluster, PUFA intake and blood lipids in children: results from the GINIplus and LISAplus studies. PLoS One. 2012;7(5):e37780. doi:10.1371/ journal.pone.0037780.

27. Hellstrand S, Sonestedt E, Ericson U, et al. Intake levels of dietary long-chain PUFAs modify the association between genetic variation in FADS and LDL-C. J Lipid Res. 2012;53(6):1183-9. doi:10.1194/ jlr.P023721.

28. • Zhang X, Qi Q, Bray GA, Hu FB, Sacks FM, Qi L. APOA5 genotype modulates 2-y changes in lipid profile in response to weight-loss diet intervention: the Pounds Lost Trial. Am J Clin Nutr. 2012;96(4):917-22. doi:10.3945/ajen.112.040907. This article reports on a fairly robust interaction between a variation implicated in several lipid parameters with diet.

29. Yap RW, Shidoji Y, Hon WM, Masaki M. Association and interaction between dietary pattern and VEGF receptor-2 (VEGFR2) gene polymorphisms on blood lipids in Chinese Malaysian and Japanese adults. Asia Pac J Clin Nutr. 2012;21(2):302-11.

30. Pollin TI, Isakova T, Jablonski KA, et al. Genetic modulation of lipid profiles following lifestyle modification or metformin treatment: the Diabetes Prevention Program. PLoS Genet. 2012;8(8):e1002895. doi:10.1371/journal.pgen.1002895.

31. - Silver HJ, Niswender KD, Keil CD, et al. CNR1 genotype influences HDL-cholesterol response to change in dietary fat intake. PLoS One. 2012;7(5):e36166. doi:10.1371/journal.pone.0036166. This article reports on a gene $x$ diet interaction study with a gene 
chosen based on our emerging understanding of the relationship between circadian and metabolic pathways.

32.• Jans A, van Hees AM, Gjelstad IM, et al. Impact of dietary fat quantity and quality on skeletal muscle fatty acid metabolism in subjects with the metabolic syndrome. Metabolism. 2012;61(11): 1554-65. doi:10.1016/j.metabol.2012.04.003. This article reports on the examination of gene expression in relevant tissues in response to dietary fat manipulation and may provide important insights to guide studies examining influences of individual genetic variation.

33. Barth SW, Koch TC, Watzl B, Dietrich H, Will F, Bub A. Moderate effects of apple juice consumption on obesity-related markers in obese men: impact of diet-gene interaction on body fat content. Eur J Nutr. 2012;51(7):841-50. doi:10.1007/s00394-011-0264-6. 